Research Article

\title{
Genetic Algorithm-Based Computed Tomography Image Analysis for the Diagnosis and Mental Health of COVID-19 Patients in Early Low-Incidence Areas
}

\author{
Yuan Niu $\mathbb{D}^{1},{ }^{1}$ Xuejie $\mathrm{He} \mathbb{D}^{2},{ }^{2}$ Guijuan Hao $\mathbb{D}^{3},{ }^{3}$ and Liang Wang $\mathbb{D}^{4}$ \\ ${ }^{1}$ Department of Office, Xingtai People's Hospital, Xingtai 054001, Hebei, China \\ ${ }^{2}$ Department of Urology, Xingtai People's Hospital, Xingtai 054001, Hebei, China \\ ${ }^{3}$ Department of Personnel, Xingtai People's Hospital, Xingtai 054001, Hebei, China \\ ${ }^{4}$ Department of First Aid, Xingtai People's Hospital, Xingtai 054001, Hebei, China \\ Correspondence should be addressed to Yuan Niu; 2620200082@stu.cpu.edu.cn
}

Received 13 June 2021; Revised 6 July 2021; Accepted 10 August 2021; Published 25 August 2021

Academic Editor: Gustavo Ramirez

Copyright (c) 2021 Yuan Niu et al. This is an open access article distributed under the Creative Commons Attribution License, which permits unrestricted use, distribution, and reproduction in any medium, provided the original work is properly cited.

The purpose of this study was to investigate the diagnosis of patients in the early low-incidence area of coronavirus disease 2019 (COVID-19) and the mental health of staff based on genetic algorithm- (GA-) based computed tomography (CT) images. In this study, 136 COVID-19 patients admitted to our hospital were divided into a critical group ( 94 cases) and a general group (42 cases). In addition, a questionnaire was used to investigate the mental health of COVID-19 patients in early low-incidence areas, including 147 medical staff members and 213 nonmedical staff members. The effects were compared between the optimized GA template matching (OGATM) algorithm proposed in this study and traditional GATM, which were applied in CT images of COVID-19 patients. The results showed that the proposed algorithm could improve the accuracy of pneumonia detection and reduce the false-positive rate. The average age of patients in the severe group was markedly higher than that of the general group $(P<0.05)$. The number of cases with diabetes mellitus $(49.6 \%)$ from the severe group was more than that from the general group $(12.4 \%)(P<0.05)$. Lymphocyte count in patients from the severe group $\left(0.68 \pm 0.26 \times 10^{9} / \mathrm{L}\right)$ was sharply lower than that of the general group $\left(1.12 \pm 0.34 \times 10^{9} / \mathrm{L}\right)(P<0.05)$. The total T lymphocyte count in patients from the severe group reduced steeply in contrast to that of the general group $(P<0.05)$. The anxiety and depression scores of medical patients $(39.45 \pm 9.45$ points and $47.58 \pm 10.47$ points) were obviously lower than the scores of nonmedical patients (43.57 \pm 9.54 points and $52.48 \pm 10.25$ points) $(P<0.05)$. In conclusion, the elderly and staffs with diabetes mellitus were more likely to develop severe COVID-19. Moreover, the total $\mathrm{T}$ lymphocytes of COVID-19 patients were lower than their normal levels, and nonmedical staffs had more psychological stress than medical staffs.

\section{Introduction}

Since December 2019, cases of unexplained and contagious pneumonia have appeared in various countries around the world. Symptoms of COVID-19 patients include fever, fatigue, dry cough, and running nose [1]. On January 7, 2020, the virus was classified and determined to be a new type of coronavirus. The World Health Organization (WHO) on January 12, 2020 denominated this new type of coronavirus "2019-novel coronavirus (2019-nCoV)" [2]. Finally, the WHO authoritatively named the pneumonia caused by the
2019 novel coronavirus infection as "coronavirus disease 2019 (COVID-19)" on February 11, 2020 [3]. As of February 14,2020 , there were 66,375 confirmed patients, 7,775 cured patients, and 1,521 deaths with COVID-19 [4]. Due to the strong contagious nature of COVID-19, the number of infections is still rising. It is urgent to diagnose it in the early low-incidence area and research on its epidemiological, clinical, and imaging characteristics and treatment plans.

Computed tomography (CT) applies the X-rays and $\gamma$-rays together with a highly sensitive detector to perform scan around a certain part of the human body. It has the 
characteristics of fast scanning time and clear images [5]. The imaging principle is to use energy rays to scan an object and rely on projection data obtained from the outside of the object, so as to adopt a specific reconstruction algorithm to present tomographic imaging of the inside of the object in the form of a 2-dimensional image. In recent years, CT imaging has been extensively used in the medical clinical diagnosis. The injured parts of the human body are scanned by $\mathrm{CT}$, and the obtained images are analyzed to determine the disease. Therefore, CT examination has become an indispensable method for lung diagnosis in the medicine [6]. The genetic algorithm (GA) is a type of search algorithm that can optimize commonly applied complex systems. Compared with previous algorithms, it can directly adopt the coding of decision variables as the calculation object, and the applicability is used for probabilistic search without other auxiliary information. The template matching (TM) algorithm proposed by Molling et al. [7] was employed to detect the researched organ or tissue in the image. TM is actually the process of people's cognition of things. At first, they will compare the similarity of the two with their previous cognitions.

COVID-19 is a new type of infectious disease for all mankind, in which epidemic prevention and control work plays a vital role [8]. The different mental health status of COVID-19 patients directly affects the transformation of the disease condition. What is more, epidemic prevention staffs may suffer from serious negative psychology under high pressure. Therefore, the effect of optimized GATM (OGATM) was compared with traditional GATM, and they were adopted in CT images of 136 patients with COVID-19. Besides, there was an investigation into the mental health status of medical staffs and nonmedical staffs in low-incidence area with the early stage of COVID-19. It aimed to early diagnose staffs with COVID-19 and identify the influencing factors of their mental health, so as to relieve pressure in a timely manner, thereby providing a reliable reference for the diagnosis of clinical COVID-19 patients.

\section{Materials and Methods}

2.1. Basic Information. In this study, 136 medically diagnosed COVID-19 patients, namely, 85 males and 51 females, who were admitted to our hospital from December 2019 to February 2020, were selected and divided into severe group (severe + critical type) (94 cases) and general group (mild + general type) (42 cases). In addition, COVID-19 was investigated in early low-incidence areas, including 147 medical staff members and 213 nonmedical staff members. This experiment had been authorized by the medical ethics committee of the hospital, and the patients and their family members had understood the situation of this experiment and signed informed consent forms.

The criteria for inclusion were defined to include patients who were diagnosed as having COVID-19 by CT imaging examination, had the imaging data that could be retrieved, and had detailed hospitalization records in this hospital.

The criteria for exclusion were defined to include subjects who were regarded as suspected cases of COVID-19, were in close contact with the diagnosed patients but undiagnosed, only had the symptom of fever, and had unclear CT images.

2.2. Research Methods. There were collections of general statistics, clinical characteristics, length of hospitalization, epidemiological history, smoking history, hypertension, and diabetes mellitus (DM) of patients with COVID-19 from the two groups. Besides, the CT imaging data of lungs should be collected. Laboratory detection indicators included the lymphocyte count, white blood cell count, and $\mathrm{T}$ lymphocyte subgroup count. Normal reference range for detection of the number of T cell subgroups was as follows. The ranges of total $\mathrm{T}$ lymphocyte count, $\mathrm{CD} 4^{+} \mathrm{T}$ lymphocyte count, and $\mathrm{CD}^{+} \mathrm{T}$ lymphocyte count were $(648-2,215) / \mu \mathrm{L}$, $(489-1,184) / \mu \mathrm{L}$, and $(196-791) / \mu \mathrm{L}$, respectively.

A questionnaire survey was used to explore the mental health of medical and nonmedical staffs. The questionnaire involved the basic statistical data, Self-Rating Anxiety Scale (SAS), nature of work, and Self-Rating Depression Scale (SDS). There were 20 items in SAS and SDS that both were 4point scales. The scores of 20 items were accumulated to get a rough score, which was multiplied by 1.25 to get the value, and the integer part of the obtained value was taken as the standard score. If the standard score was greater than 50 , it indicated that the staffs suffered from anxiety or depression.

2.3. Diagnostic Criteria for COVID-19. According to the New Coronavirus Pneumonia Diagnosis and Treatment Plan (Trial Version 5), the diagnostic criteria were as follows. First, there was an intimate contact of people and patients with COVID-19. Second, the clinical features included fever, dryness, dry cough, and running nose. Besides, the imaging features met the criteria for pneumonia. Third, in the etiological features, the respiratory tract specimens detected by real-time fluorescent quantitative reverse transcription-polymerase chain reaction (RT-PCR) were positive for nucleic acid of COVID-19, and respiratory specimens were highly homologous to known patients with COVID-19.

Clinical classification criteria were as follows. The first one was the mild type. The clinical symptoms were mild, and CT images had no obvious features of pneumonia. The second one was the normal type: there were symptoms such as fever and cough, and the features of pneumonia could be observed on CT images. The third one was the severe type (patients were in accordance with one of the following). The oxygen saturation was less than or equal to $93 \%$ in rest state; respiratory frequency of respiratory distress (RR) was more than or equal to 30 times/min; and arterial oxygen partial pressure $\left(\mathrm{PaO}_{2}\right) /$ inspired oxygen concentration $\left(\mathrm{FiO}_{2}\right)$ was equal to or less than $300 \mathrm{mmHg}$. The fourth one was the critical type (patients had one of the following). There was respiratory failure or shock; mechanical ventilation was required; and patients suffered from other organ failures and needed monitoring and treatment of intensive care unit (ICU). 
2.4. CT Scanning Examination of All Patients. The GE LightSpeed VCT 64-slice spiral CT scanner was used in this study, and patients should be instructed to remove metal jewelry on the chest before scanning. Besides, they needed to test for iodine allergy in advance; the scanning process should be explained to make patients maintain a calm state of mind. First, the chest underwent a left and right plain scanning, and then patients were scanned from the top to bottom. The scan range was from the upper thorax to the diaphragm, and then an enhanced scan was adopted. The scanning layer thickness was $0.625 \mathrm{~mm}$, the scanning interval was $0.5 \mathrm{~mm}$, the pitch was $0.984: 1$, the matrix was $512 \times 512$, the voltage was $120 \mathrm{kV}$, and the current was 220-500 mA. The bolus injection method was employed to inject the contrast agent. Firstly, $15 \mathrm{~mL}$ of normal saline was injected from the vein to check whether there was a subcutaneous leakage. The injection rate of contrast agent iopromide injection was $3.5 \mathrm{~mL} / \mathrm{s}$, and the injection volume was $80 \mathrm{~mL}$. Then, $35 \mathrm{~mL}$ of normal saline was injected in the same way, thereby reducing the concentration of contrast agent in the superior vena cava. After the contrast agent injection, the scan would be conducted with a delay of 5 seconds when its concentration reached the threshold.

\subsection{Calculation Steps of Template Matching Genetic} Algorithm. The basic flowchart of GA is shown in Figure 1. Similarity measures included the correlation coefficient, moment invariant, and sequence similarity test. The correlation coefficient referred to the correlation between the template $S$ and the image $g$. Assuming that $N=2^{l}$, the following equation could be satisfied.

$$
T(p, q)=\frac{\sum_{x=1}^{N} \sum_{y=1}^{N} S(x, y) g(p-(N / 2)+x, q-(N / 2)+y)}{\left[\sum_{x=1}^{N} \sum_{y=1}^{N} S^{2}(x, y)\right]^{1 / 2}} q .
$$

In equation (1), $T(p, q)$ represents the correlation coefficient between the template and the image, the template size is $N \times N$, the coordinate point of the template $T(x, y)$ is $(x, y)$, and $(p, q)$ stands for the coordinates in the image. The invariant moment measurement was the quantification of the eigenvalues of the gray distribution of the template and the measured image. In addition, the definition equation of the matrix of the 2-dimensional continuous function was as follows:

$$
n(a, b)=\int_{-\infty}^{\infty} \int_{-\infty}^{\infty} x^{a} y^{b} g(x, y) \mathrm{d} x \mathrm{~d} y .
$$

In equation (2), $g(x, y)$ stands for a two-dimensional continuous function and $n(a, b)$ represents a matrix of $a, b$. There was a discretization when satisfying $a=0,1,2, \ldots$ and $b=0,1,2, \ldots$. What is more, the numbers in the 2 -dimensional image could be converted into a density distribution function, and the summation equation was adopted instead of the integral function, so the following equation could be obtained:

$$
n_{a, b}=\sum_{o} \sum_{p} o^{a} p^{b} g(o, p) .
$$

In equation (3), $g(o, p)$ expresses a two-dimensional continuous function that satisfies $a=0,1,2, \ldots$ and $b=0,1,2, \ldots$ for discretization. In order to keep the displacement of the image constant, a central matrix of $a+b$ order was adopted, which could be expressed as follows:

$$
\delta_{a b}=\sum_{o} \sum_{p}(o-\bar{o})^{a}(p-\bar{p})^{b} g(o, p) .
$$

In equations (3) and (4), $\bar{o}=n_{10} / n_{00}$ and $\bar{p}=n_{01} / n_{00}$ should be satisfied.

When the invariant moment was applied, the first-order moments of the template and the measured image were calculated, respectively, and then the sizes of the two needed to be compared. The sequence similarity between the template and the image should be simplified to the absolute difference between the two. If the template size was $N=2^{l}$, the equation of absolute difference measurement $R(p, q)$ was as follows:

$$
R(p, q)=\sum_{x=1}^{N} \sum_{y=1}^{N}\left|S(x, y) g\left(p-\left(\frac{N}{2}\right)+x, q-\left(\frac{N}{2}\right)+y\right)\right| .
$$

In equation (5), the matching degree between the two was higher if the $R$ value was smaller. Moreover, the two were completely consistent if the $R$ value was equal to 0 . The Fisher linear discriminant analysis (LDA) classifier was often applied, and the linear discriminant function equation could be expressed as follows:

$$
b(\vec{x})=\theta_{1} x_{1}+\theta_{2} x_{2}+\cdots+\theta_{n} x_{n}+\theta_{n+1} x_{n+1} .
$$

In equation (6), the $n$-dimensional measurement mode is $\vec{x}_{1}, \vec{x}_{2}, \ldots, \vec{x}_{n}$, and the $n_{1}$ and $n_{2}=n-n_{1}$ modes both belong to $\theta_{1}$ and $\theta_{2}$, respectively. The average value of various modes could be calculated as follows:

$$
n_{j}=\frac{1}{M} \sum_{i} y_{i}^{(j)}, \quad i, j=1,2 .
$$

In equation (7), various modes are generally denoted as $\left\{\vec{y}_{i}^{(j)}\right\}$ and $n_{j}$ stands for the mean vector. The inward-off matrix of various classes is expressed as

$$
R_{V_{a}}=\sum_{b}\left(x_{b}^{(a)}-n_{a}\right)\left(x_{b}^{(a)}-n_{b}\right), \quad a, b=1,2 .
$$

The following equation shows the total in-class distance matrix $R_{V}$ :

$$
R_{V}=R_{V_{1}}+R_{V_{2}}
$$

Then, the class distance matrix $R_{C}$ is shown as follows:

$$
R_{C}=\left(n_{1}-n_{2}\right)\left(n_{1}-n_{2}\right)^{\prime} .
$$

The $n$-dimensional vector $\vec{x}$ was mapped in the vector $\vec{w}$ direction, which could be expressed in 


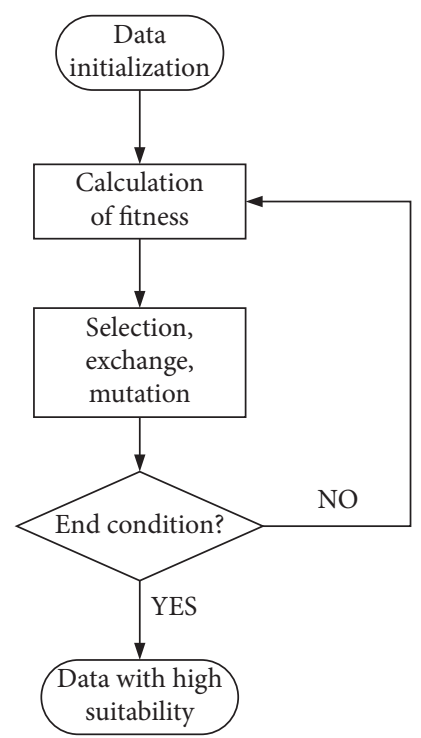

Figure 1: Basic flowchart of GA.

$$
x_{b}^{(a)}=v^{\prime} x_{b}^{(a)}
$$

After the exchange, the mean expression of various modes in the $x$ space could be indicated in

$$
\tilde{n}_{a}=\frac{1}{M_{a}} \sum_{b} x_{b}^{(a)}, \quad a=1,2 .
$$
$\widetilde{R}_{V_{a}}^{2}$ :

The following equation shows within-class deviation

$$
\widetilde{R}_{V_{a}}^{2}=\sum_{b}\left(x_{b}^{(a)}-\tilde{n}_{a}\right)^{2}, \quad a, b=1,2 .
$$

The amount of deviation $\widetilde{R}_{V}^{2}$ within the total class could be calculated as

$$
\widetilde{R}_{V}^{2}=\widetilde{R}_{V_{a}}^{2}+\widetilde{R}_{V_{b}}^{2}, \quad a, b=1,2 .
$$

The deviation $\widetilde{R}_{C}^{2}$ between classes is shown in

$$
\widetilde{R}_{C}^{2}=\left(\widetilde{n}_{1}-\widetilde{n}_{2}\right)^{2}
$$

After mapping, it was necessary to gather similar patterns together and disperse different types of patterns. Therefore, $\widetilde{R}_{V}^{2}$ needed to be as small as possible and $\widetilde{R}_{C}^{2}$ as large as possible, and the following functions were required as benchmarks:

$$
S_{E}(\vec{w})=\frac{\vec{w}^{\prime} R_{C} \vec{w}}{\vec{w}^{\prime} R_{v} \vec{w}} .
$$

Equation (16) is called Fisher's linear discriminant equation. In order to make $S_{E}(\vec{w})$ get the maximum value, the equation could be obtained according to the derivation of $S_{E}$ to $\vec{w}$.

$$
\vec{w}=\frac{\beta}{\mu} R_{E}^{-1}\left(\vec{n}_{1}-\vec{n}_{2}\right)
$$

In equation (17), $\beta / \mu$ represents a quantitative factor. If the direction of the vector was not changed, the value was set to 1 , and the following equation was obtained:

$$
\vec{w}=R_{E}^{-1}\left(\vec{n}_{1}-\vec{n}_{2}\right) .
$$

Since the 1-dimensional model was obtained after the exchange, it was determined that the interface was different coordinate points on the axis in various modes.

2.6. Statistical Methods. In this study, SPSS 22.0 statistical software was used for analysis. The data that met the normal distribution were expressed as the mean \pm standard deviation $(\bar{x} \pm s)$, and the independent sample $t$-test was used for group comparison between groups, anxiety score, and depression score. The frequency and percentage were employed to express the nonnormal distribution data, the detection and false-positive rates of TM and GATM, the proportion of general statistical data of patients, and lymphocyte count. The multiple linear regression was adopted to gradually analyze the influencing factors of anxiety and depression of medical and nonmedical staffs. $P<0.05$ showed that the difference was statistically substantial.

\section{Results}

3.1. Analysis of Simulation Effects of the Two Algorithms. The proposed algorithm was compared with the traditional GATM, both of which were employed to the CT images of 136 COVID-19 patients. As shown in Figure 2, the detection rate of the proposed algorithm was obviously improved $(P<0.05)$. Besides, its false-positive rate averagely detected decreased sharply, with a statistically obvious difference $(P<0.05)$. This showed that the proposed algorithm could greatly promote the accuracy of pneumonia detection and reduce the false-positive rate of pneumonia.

3.2. General Data of COVID-19 Patients. The difference in the gender was not statistically marked among patients in both groups $(P>0.05)$. The average age of patients from the severe group was obviously higher than the age of the general group $(P<0.05)$. There was no marked difference in smoking history of patients from the severe group $(10.2 \%)$ and the general group (6.8\%), and the difference was not statistically obvious $(P>0.05)$ (Figure 3$)$. The proportion of cases with hypertension from the severe group (9.3\%) was not statistically different from that of the general group $(8.6 \%)(P>0.05)$. In addition, Figure 4 indicates that the proportion of patients with diabetes mellitus from the severe group $(49.6 \%)$ was dramatically higher than the proportion of the general group $(12.4 \%)(P<0.05)$.

3.3. Clinical Characteristics of COVID-19 Patients. There were a total of 136 patients, and fever was the most common clinical symptom. Other symptoms included cough, fatigue, muscle soreness, expectoration, running nose, head dizziness, nausea and vomiting, chest tightness, and shortness of breath. 9 patients had no obvious clinical manifestations before admission. After admission, the above manifestations gradually appeared, and the CT images also gradually 


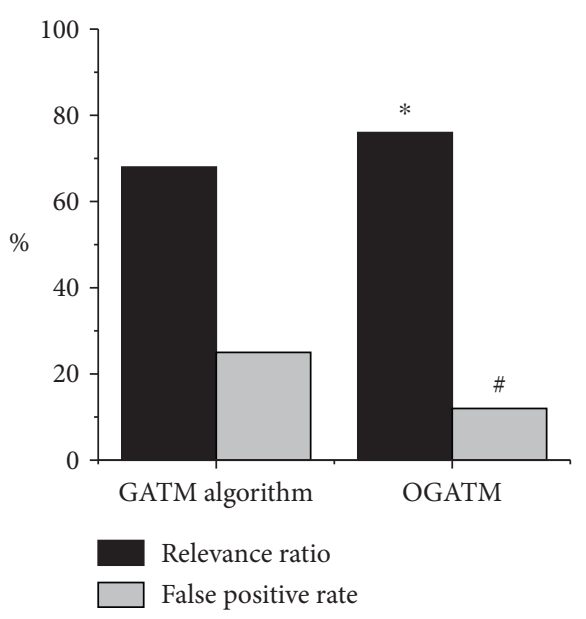

Figure 2: Simulation effect analysis of the two algorithms. (Note: ${ }^{*}$ means that there was a statistically substantial difference in contrast to the detection rate of GATM algorithm $(P<0.05)$; ${ }^{*}$ indicates that the difference was statistically marked in contrast to the false-positive rate of GATM algorithm $(P<0.05))$.

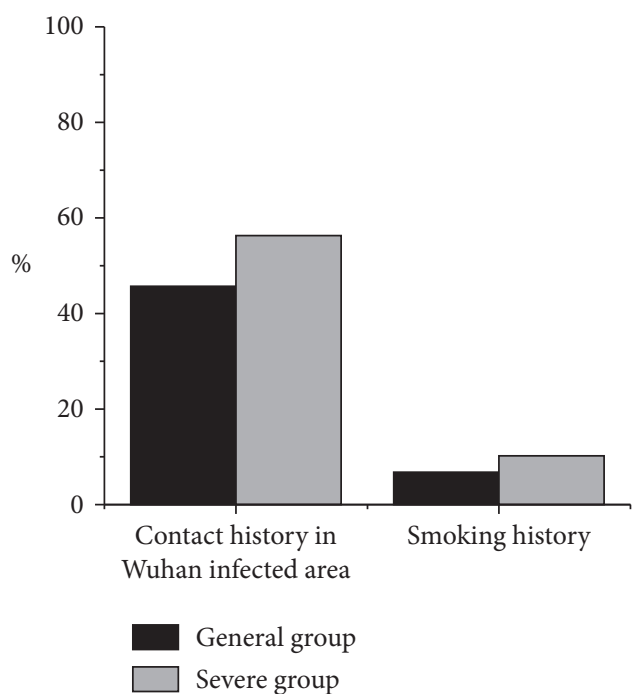

FIgURE 3: Comparison of the epidemiological and smoking histories among patients from the two groups.

showed the characteristics of pneumonia. In addition, the number of cases with symptoms such as cough, fatigue, and sputum from the severe group was greatly higher than that from the general group, and the difference was statistically remarkable $(P<0.05)$ (Figures 5 and 6$)$.

\subsection{CT Imaging Characteristics of Patients with COVID-19.} The CT imaging at the very early stage (no obvious clinical symptoms) was mainly manifested as a single ground glass shadow or a few fiber streaks. In the early stage (0-4 days after the initial symptoms), the main manifestations were scattered ground glass shadows, the main location was near the pleura of one or both lung lobes, and there was no obvious abnormality in both lungs. The CT imaging of early patients presented single or multiple ground glass shadows, and there were nodular, patchy, or lamellar shadows, with or without thickening of the interlobular septum. The CT imaging at the advanced stage (5-8 days after the initial symptoms) mainly showed the diffuse ground glass shadow and "paving stone" sign, and its main location was bilateral multiple lung lobes. The CT imaging at the severe stage (9-13 days after the initial symptoms) was mainly manifested as lung consolidation, with main location of diffused lungs (Figure 7). As the disease progressed, the consolidation area began to be absorbed, the density decreased, and it gradually became a ground glass density shadow (Figure 8), just like a sugar melted. Besides, the effective treatment or the body's immunity could gradually overcome the virus, the inflammation would be further absorbed, and the scope would gradually shrink; maybe a fiber strip stave was left.

3.5. Auxiliary Examinations for COVID-19 Patients. After admission, the difference in the white blood cell count was not statistically great among patients from both groups $(P>0.05)$. The lymphocyte counts in patients from the severe group $\left(0.68 \pm 0.26 \times 10^{9} / \mathrm{L}\right)$ were sharply lower than the counts of the general group $\left(1.12 \pm 0.34 \times 10^{9} / \mathrm{L}\right)$, with a statistically substantial difference $(P<0.05)$ (Figure 9$)$. The total $\mathrm{T}$ lymphocyte count and $\mathrm{CD} 4^{+}$and $\mathrm{CD}{ }^{+} \mathrm{T}$ lymphocyte count of patients from the severe group reduced sharply in contrast to those of the general group, and there was a statistically obvious difference $(P<0.05)$ (Figure 10).

3.6. Mental Health of Staffs in the Early Low-Incidence Areas of COVID-19. Under the circumstance of ensuring that other factors were the same, there was a comparison of the SAS and SDS scores of medical and nonmedical staffs in areas with low incidence of COVID-19. The results revealed that the anxiety and depression scores of medical staffs $(39.45 \pm 9.45$ points and $47.58 \pm 10.47$ points) decreased steeply in contrast to those of nonmedical staffs $(43.57 \pm 9.54$ points and $52.48 \pm 10.25$ points) $(P<0.05)$ (Figure 11$)$. Besides, Figure 12 shows that there was a multiple linear regression analysis of anxiety symptoms for people with an annual family income of 50,000-100,000 and those with an annual family income of 100,000-200,000. It indicates that the anxiety symptoms of staffs with a yearly family income of 100,000-200,000 were more evident $(t=2.3)$, and there was a statistically huge difference $(P<0.05)$.

\section{Discussion}

The new type of coronavirus is a $\beta$-type coronavirus. Up till now, most of the medical understanding of coronaviruses stems from the research on severe acute respiratory syndrome (SARS)-CoV [9]. In this study, the average age of patients from the severe group was markedly higher than that of the general group, which might be related to the patients' immunity. The elderly had low resistance and were prone to develop severe disease after being infected with the COVID-19, which was consistent with the research results of $\mathrm{Xu}$ et al. [10]. Aggarwal et al. [11] pointed out that the new type of coronary pneumonia with multiple diseases was 


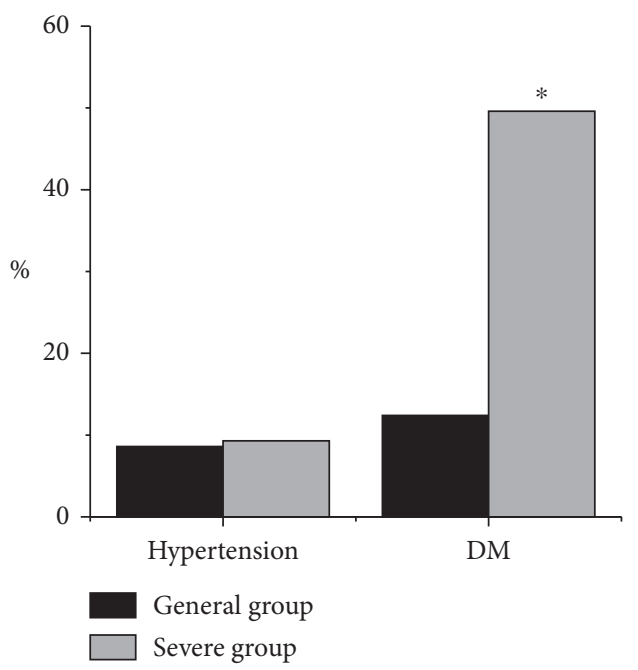

FIGURE 4: Comparison of the proportion of patients with hypertension and DM in the two groups. (Note: ${ }^{*}$ indicates that there was a statistically obvious difference in contrast to the general group $(P<0.05))$.

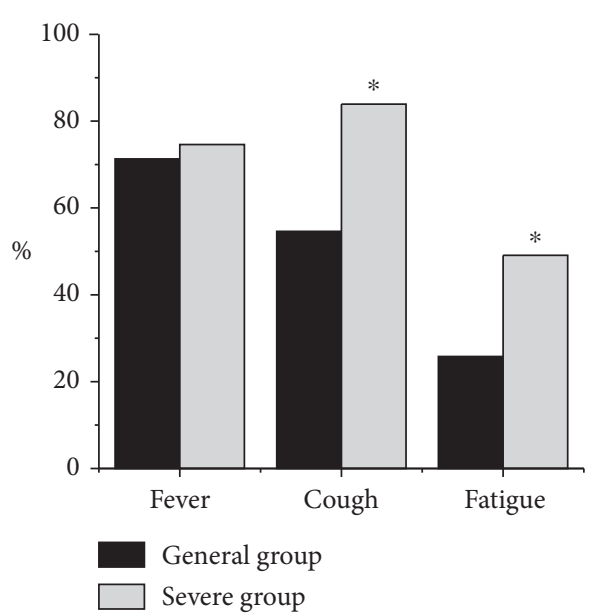

Figure 5: Comparison of the number of cases with fever, cough, and fatigue in the two groups. (Note: ${ }^{*}$ shows that there was a statistically substantial difference in contrast to the general group $(P<0.05))$.

more likely to develop into severe and critical illness. The patients with hypertension and DM in the general and severe groups were compared in this study, and it was found that patients with DM were more likely to become severely ill, with a statistically obvious difference $(P<0.05)$. The above research was supplemented, which indicated that DM led to a decrease in immunity of patients and an increase in the chance of serious respiratory infections.

Looking at the epidemiological history of COVID-19, second-generation and third-generation confirmed cases are still increasing over time. Moreover, it indicated that COVID19 was very contagious and should be timely diagnosed and treated for early patients, but the difference between the two groups was not statistically remarkable $(P>0.05)$. These results were in line with the research findings of Ahn et al. [12].

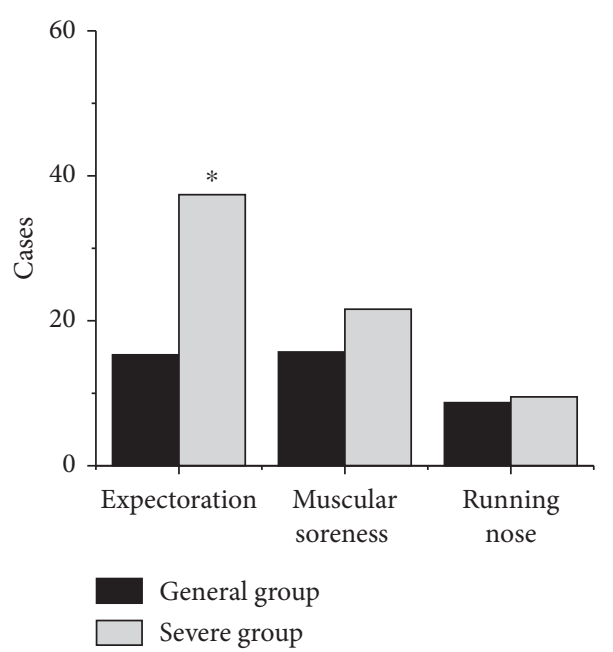

FIgURE 6: Comparison of the number of patients with expectoration, muscle soreness, and running nose in the two groups. (Note: * shows that the difference was statistically substantial in contrast to the general group $(P<0.05))$.

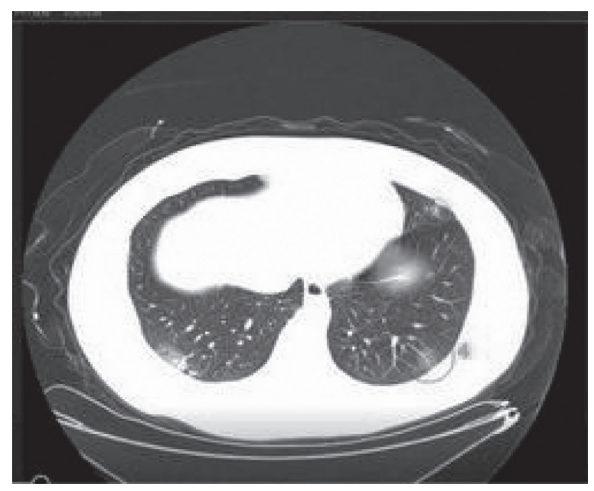

Figure 7: A CT image of one male patient with COVID-19 (48 years old). (Note: the patients suffered from fever and cough on the $9^{\text {th }}$ day after returning to hometown from Wuhan, and CT image showed solid nodules).

Current epidemiological research had pointed out that the incubation period of COVID-19 is 2 weeks, and the main clinical manifestations are fever, cough, fatigue, and muscle soreness. Severely ill patients will experience severe respiratory disorders after about 7 days of illness [13]. The clinical characteristics of this study are consistent with the above research, mainly fever, cough, and sputum. In the study, 9 patients had no obvious clinical manifestations before admission. After admission, the above manifestations gradually appeared, and the CT images gradually showed the characteristics of pneumonia. This reveals that most early COVID-19 patients have marked clinical features, but there are still a small number of patients who have no obvious symptoms.

In this study, the number of $\mathrm{T}$ lymphocytes, $\mathrm{CD} 4{ }^{+}$ $\mathrm{T}$ lymphocytes, and $\mathrm{CD} 8^{+} \mathrm{T}$ lymphocytes in patients from the severe group reduced steeply compared with the general group, suggesting that COVID-19 would actively attack the human immune system after entering the human body, 


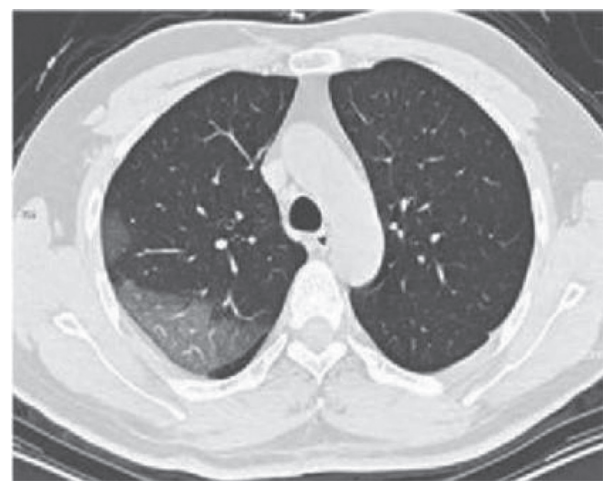

Figure 8: A CT image of one male patient with COVID-19 (53 years old). (Note: he developed fever with $38.3^{\circ} \mathrm{C}$ on the 8 th day after returning home from Wuhan, and CT image showed central consolidation that was around ground glass).

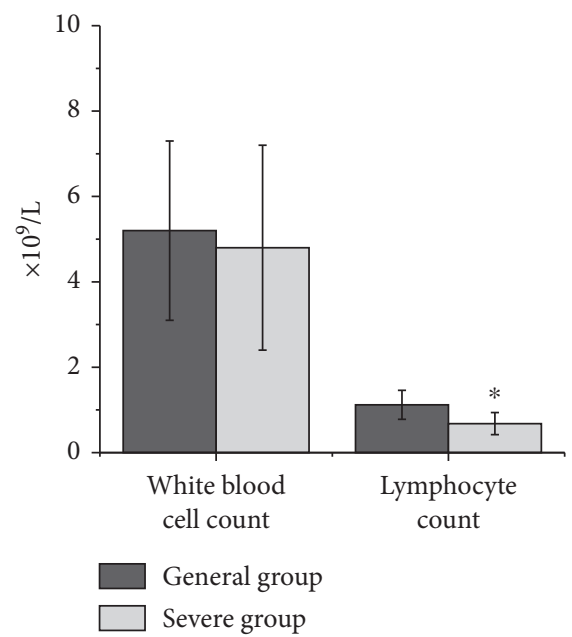

Figure 9: Comparison of the white blood cell count and lymphocyte count of patients from the two groups. (Note: * shows that the difference was statistically marked in contrast to the general group $(P<0.05))$.

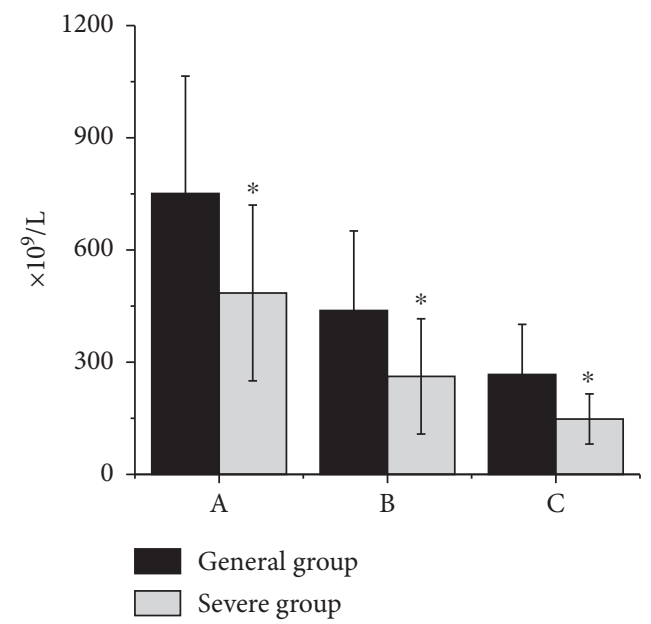

FIGURE 10: Comparison of lymphocyte counts of patients in the two groups. (Note: A, B, and C stand for the total T lymphocyte count, CD $4^{+}$ $\mathrm{T}$ lymphocyte count, and $\mathrm{CD} 8^{+} \mathrm{T}$ lymphocyte count, respectively. ${ }^{*}$ indicates that there was a statistically marked difference in contrast to the general group $(P<0.05))$.

resulting in the drop of the number of immune cells. When the lymphocyte content decreased in the early stage, the current patients were injected with immunoglobulin to gradually improve the patients' condition that was transferred to the normal type until the patients were discharged. Therefore, early monitoring of the number of 


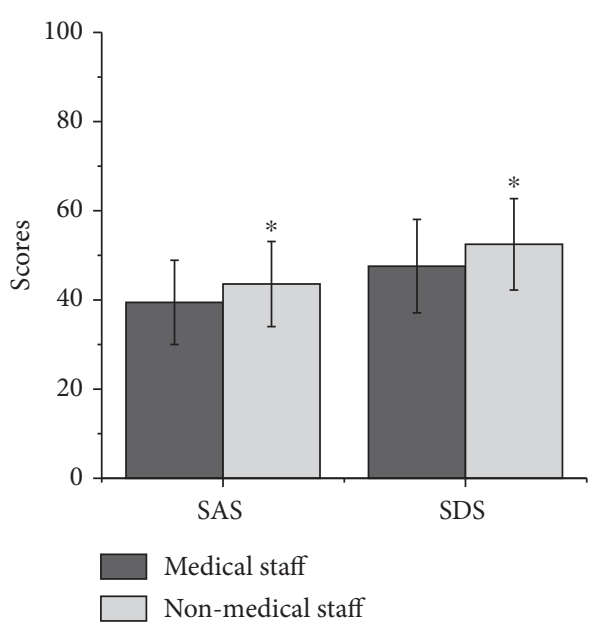

FIgURE 11: Comparison of the SAS and SDS scores between medical and nonmedical staffs. (Note: ${ }^{*}$ indicates that there was a statistically huge difference in contrast to the general group $(P<0.05))$.

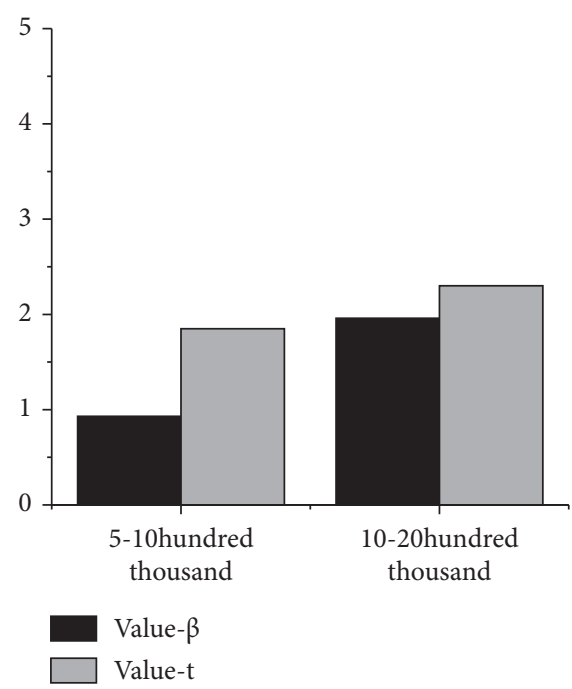

Figure 12: Multiple linear regression analysis of the per capita annual income of medical and nonmedical staffs.

$\mathrm{T}$ lymphocytes, $\mathrm{CD} 4^{+} \mathrm{T}$ lymphocytes, and $\mathrm{CD} 8^{+}$ T lymphocytes in COVID-19 patients was helpful for timely clinical diagnosis and corresponding treatment measures to reduce the mortality of COVID-19 patients. This was in accordance with the results of Liu et al. [14] and Peng et al. [15].

The anxiety and depression scores of medical and nonmedical staffs in this study were substantially higher than those reported by first-line epidemic prevention staffs in Chongqing [16]. The anxiety and depression scores of nonmedical staffs were both higher than those of medical staffs in this study. This might be due to the lack of publicity about infectious diseases, which made nonmedical staffs lack knowledge of COVID-19. In addition, the high-intensity epidemic prevention measures grew their psychological pressure.

\section{Conclusion}

The effect of the algorithm proposed in this study was compared with the effect of the traditional GATM, and both of them were adopted in the CT images of 136 patients with COVID-19. It was found that the TM algorithm could greatly improve the accuracy of pneumonia detection and reduce the false-positive rate of pneumonia. The clinical characteristics of COVID-19 patients mainly included fever, cough, muscle soreness, sputum expectoration, and fatigue. What is more, the elderly patients with DM were more likely to develop severe illness. The total $\mathrm{T}$ lymphocyte count, $\mathrm{CD} 4^{+} \mathrm{T}$ lymphocyte count, and $\mathrm{CD} 8^{+} \mathrm{T}$ lymphocyte count in COVID-19 severe patients were lower than their normal levels. Nonmedical staffs had higher anxiety and depression scores than medical staffs, with excessive psychological pressure. The deficiency of this study was that the sample size was small (in particular, the number of critically ill patients was low), which may lead to certain deviations in the research results. Later, it is necessary to consider increasing the sample range for further research. To sum up, the results of this study not only contributed to the clinical diagnosis of early COVID-19, but also paid close attention to the mental health of epidemic prevention staffs in high-intensity work, so as to maximize the efficiency of epidemic prevention and control work.

\section{Data Availability}

The data used to support the findings of this study are available from the corresponding author upon request.

\section{Conflicts of Interest}

The authors declare no conflicts of interest.

\section{References}

[1] X.-W. Xu, X.-X. Wu, X.-G. Jiang et al., "Clinical findings in a group of patients infected with the 2019 novel coronavirus (SARS-Cov-2) outside of Wuhan, China: retrospective case series," BMJ, vol. 368, p. m606, 2020.

[2] Y. H. Jin, L. Cai, and Z. S. Cheng, "A rapid advice guideline for the diagnosis and treatment of 2019 novel coronavirus (2019$\mathrm{nCoV}$ ) infected pneumonia (standard version)," Military Medical Research, vol. 7, no. 1, p. 4, 2020.

[3] C.-C. Lai, C.-Y. Wang, Y.-H. Wang, S.-C. Hsueh, W.-C. Ko, and P.-R. Hsueh, "Global epidemiology of coronavirus disease 2019 (COVID-19): disease incidence, daily cumulative index, mortality, and their association with country healthcare resources and economic status," International Journal of Antimicrobial Agents, vol. 55, no. 4, Article ID 105946, 2020.

[4] S. A. Lauer, K. H. Grantz, Q. Bi et al., "The incubation period of coronavirus disease 2019 (COVID-19) from publicly reported confirmed cases: estimation and application," Annals of Internal Medicine, vol. 172, no. 9, pp. 577-582, 2020.

[5] A. Parakh, F. Macri, and D. Sahani, "Dual-energy computed tomography," Radiologic Clinics of North America, vol. 56, no. 4, pp. 601-624, 2018.

[6] D. Chiumello, A. Marino, M. Brioni et al., "Lung recruitment assessed by respiratory mechanics and computed tomography in patients with acute respiratory distress syndrome. what is 
the relationship?" American Journal of Respiratory and Critical Care Medicine, vol. 193, no. 11, pp. 1254-1263, 2016.

[7] D. Molling, B. M. Vincent, W. L. Wiitala et al., "Developing a template matching algorithm for benchmarking hospital performance in a diverse, integrated healthcare system," Medicine, vol. 99, no. 24, Article ID e20385, 2020.

[8] H. Luo, Q.-1. Tang, Y.-X. Shang et al., "Can Chinese medicine be used for prevention of corona virus disease 2019 (COVID19)? A review of historical classics, research evidence and current prevention programs," Chinese Journal of Integrative Medicine, vol. 26, no. 4, pp. 243-250, 2020.

[9] L. Chen, W. Liu, Q. Zhang et al., "RNA based MNGS approach identifies a novel human coronavirus from two individual pneumonia cases in 2019 Wuhan outbreak," Emerging Microbes \& Infections, vol. 9, no. 1, pp. 313-319, 2020.

[10] X. Xu, C. Yu, J. Qu et al., "Imaging and clinical features of patients with 2019 novel coronavirus SARS-CoV-2," European Journal of Nuclear Medicine and Molecular Imaging, vol. 47, no. 5, pp. 1275-1280, 2020.

[11] G. Aggarwal, G. Lippi, C. J. Lavie, B. M. Henry, and F. Sanchis-Gomar, "Diabetes mellitus association with coronavirus disease 2019 (COVID-19) severity and mortality: a pooled analysis," Journal of Diabetes, vol. 12, no. 11, pp. 851-855, 2020.

[12] D.-G. Ahn, H.-J. Shin, M.-H. Kim et al., "Current status of epidemiology, diagnosis, therapeutics, and vaccines for novel coronavirus disease 2019 (COVID-19)," Journal of Microbiology and Biotechnology, vol. 30, no. 3, pp. 313-324, 2020.

[13] Y. R. Guo, Q. D. Cao, Z. S. Hong et al., "The origin, transmission and clinical therapies on coronavirus disease 2019 (COVID-19) outbreak-an update on the status," Military Medical Research, vol. 7, no. 1, p. 11, 2020.

[14] J. Liu, S. Li, J. Liu et al., "Longitudinal characteristics of lymphocyte responses and cytokine profiles in the peripheral blood of SARS-CoV-2 infected patients," EBioMedicine, vol. 55, Article ID 102763, 2020.

[15] Y. Peng, A. J. Mentzer, A. J. Mentzer et al., "Broad and strong memory CD4+ and CD8+ T cells induced by SARS-CoV-2 in UK convalescent individuals following COVID-19," Nature Immunology, vol. 21, no. 11, pp. 1336-1345, 2020.

[16] L. Lei, X. Huang, S. Zhang, J. Yang, L. Yang, and M. Xu, "Comparison of prevalence and associated factors of anxiety and depression among people affected by versus people unaffected by quarantine during the COVID-19 epidemic in southwestern China," Medical Science Monitor: International Medical Journal of Experimental and Clinical Research, vol. 26, Article ID e924609, 2020. 